\title{
Descemet stripping automated endothelial keratoplasty using corneas from elderly donors
}

\author{
Parul Chawla Gupta ${ }^{1}$ Jagat Ram ${ }^{1}$
}

Received: 29 June 2016 / Accepted: 29 August 2016 / Published online: 10 September 2016

(C) Springer-Verlag Berlin Heidelberg 2016

\section{Dear Editor,}

We read with interest the recent paper by Nakatani et al. [1] evaluating the outcomes of Descemet stripping automated endothelial keratoplasty (DSAEK) using corneas from elderly donors. While the study is indeed interesting, there are certain points we wish to highlight. First, according to the study, the rate of endothelial cell loss at 2 years after surgery was similar regardless of donor age ( $\geq 80$ vs. $\leq 79$ years). Donor age and diabetic status have been identified as factors affecting corneal endothelial cell count. With every year increase in age (after adjusting for diabetic status), the cell count decreases by 6.4 cells [95\% confidence interval (CI), -8.3 to -4.5 ]; likewise, in those with diabetes (after adjusting for age), the cell count is lower by 66 cells (95\% CI, 6.3-125.9) compared with controls [2]. However, there is no significant difference in the endothelial count between groups in the present study; we believe that corneas from donors $\geq 80$ years over a longer period of followup might eventually have a poorer endothelial cell count. Moreover, we wonder whether the authors took into consideration the presence or absence of diabetes mellitus in the two groups, since a higher prevalence of diabetes in group 2 could have reduced the endothelial count despite having included younger donors. Second, cigarette smoking reduces endothelial cell count [3]. Smoking history should also have been considered in comparing the endothelial counts between groups. Moreover, advanced nuclear cataract and chronic pulmonary

Jagat Ram

drjagatram@gmail.com

1 Department of Ophthalmology, Post Graduate Institute of Medical Education and Research, Chandigarh, India 160012 disease are significant risk factors for reduced endothelial density. Although the mechanisms are unknown, patients with these risk factors may have a poor endothelial reserve [4]. The authors should therefore rule out all the aforementioned factors before analyzing the results.

\section{Compliance with ethical standards}

Funding No funding was received for this research.

Conflict of interest All authors certify that they have no affiliations with or involvement in any organization or entity with any financial interest (such as honoraria; educational grants; participation in speakers' bureaus; membership, employment, consultancies, stock ownership, or other equity interest; and expert testimony or patent-licensing arrangements) or non-financial interest (such as personal or professional relationships, affiliations, knowledge or beliefs) in the subject matter or materials discussed in this manuscript.

\section{References}

1. Nakatani S, Murakami A (2016) Descemet stripping automated endothelial keratoplasty using corneas from elderly donors. Graefes Arch Clin Exp Ophthalmol 254:1135-1140

2. Sudhir RR, Raman R, Sharma T (2012) Changes in the corneal endothelial cell density and morphology in patients with type 2 diabetes mellitus: a population based study, Sankara Nethralaya Diabetic Retinopathy and Molecular Genetics Study (SNDREAMS, Report 23). Cornea 1-4

3. Ilhan N, Ilhan O, Coskun $\mathrm{M}$ et al (2015) Effects of smoking on central corneal thickness and the corneal endothelial cell layer in otherwise healthy subjects. Eye Contact Lens. doi:10.1097 /ICL.0000000000000212

4. Ishikawa A (2002) Risk factors for reduced corneal endothelial cell density before cataract surgery. J Cataract Refract Surg 28(11):1982-1992 